\title{
Molecular characteristics of Human Endogenous Retrovirus type-W in schizophrenia and bipolar disorder
}

\author{
H Perron ${ }^{1,2,3}$, N Hamdani ${ }^{4,5,6,7}$, R Faucard ${ }^{2}$, M Lajnef ${ }^{4,5,6}$, S Jamain ${ }^{4,6}$, C Daban-Huard ${ }^{4,5,6}$, S Sarrazin ${ }^{4,5,6,8}$, E LeGuen Len, $^{4,5,6}$, \\ $\mathrm{J} \mathrm{Houenou}^{4,5,6,8}$, M Delavest ${ }^{6,9}{ }^{6}$, H Moins-Teiserenc ${ }^{10}$, D Bengoufa ${ }^{10}$, R Yolken ${ }^{11}$, A Madeira ${ }^{2}$, M Garcia-Montojo ${ }^{2}$, N Gehin ${ }^{2}$, I Burgelin ${ }^{2}$, \\ G Ollagnier $^{2}$, C Bernard ${ }^{1}$, A Dumaine ${ }^{4,6}$, A Henrion ${ }^{4,6}$, A Gombert ${ }^{4,6}$, K Le Dudal ${ }^{12,14,15}$, D Charron ${ }^{10}$, R Krishnamoorthy ${ }^{13}$, \\ R Tamouza ${ }^{10,16}$ and M Leboyer ${ }^{4,5,6,7,16}$
}

Epidemiological and genome-wide association studies of severe psychiatric disorders such as schizophrenia (SZ) and bipolar disorder (BD), suggest complex interactions between multiple genetic elements and environmental factors. The involvement of genetic elements such as Human Endogenous Retroviruses type 'W' family (HERV-W) has consistently been associated with SZ. HERV-W envelope gene (env) is activated by environmental factors and encodes a protein displaying inflammation and neurotoxicity. The present study addressed the molecular characteristics of HERV-W env in SZ and BD. Hundred and thirty-six patients, 91 with BD, 45 with SZ and 73 healthy controls (HC) were included. HERV-W env transcription was found to be elevated in $\mathrm{BD}(P<10-4)$ and in $\mathrm{SZ}(P=0.012)$ as compared with $\mathrm{HC}$, but with higher values in $\mathrm{BD}$ than in $\mathrm{SZ}$ group $(P<0.01)$. The corresponding DNA copy number was paradoxically lower in the genome of patients with $\mathrm{BD}(P=0.0016)$ or $\mathrm{SZ}(P<0.0003)$ than in HC. Differences in nucleotide sequence of HERV-W env were found between patients with SZ and BD as compared with HC, as well as between SZ and BD. The molecular characteristics of HERV-W env also differ from what was observed in Multiple Sclerosis (MS) and may represent distinct features of the genome of patients with BD and SZ. The seroprevalence for Toxoplasma gondii yielded low but significant association with HERV-W transcriptional level in a subgroup of BD and SZ, suggesting a potential role in particular patients. A global hypothesis of mechanisms inducing such major psychoses is discussed, placing HERV-W at the crossroads between environmental, genetic and immunological factors. Thus, particular infections would act as activators of HERV-W elements in earliest life, resulting in the production of an HERV-W envelope protein, which then stimulates pro-inflammatory and neurotoxic cascades. This hypothesis needs to be further explored as it may yield major changes in our understanding and treatment of severe psychotic disorders.

Translational Psychiatry (2012) 2, e201; doi:10.1038/tp.2012.125; published online 4 December 2012

\section{Introduction}

Schizophrenia (SZ) and bipolar disorder (BD) are severe psychiatric disorders involving complex interactions between genetic and environmental factors. ${ }^{1,2}$ Environmental factors, such as winter birth, urban environment and maternal infection during pregnancy, in particular caused by Influenza virus, Herpesviruses or $T$. gondii, are associated with an increased risk for $\mathrm{SZ}$ and for $\mathrm{BD} .^{3-5}$ Particular viruses or parasites have been thought to have a role in the pathogenesis of SZ or BD but, as most studies were based on serology that essentially detects an 'immunological scar' represented by the presence of specific immunoglobulin $G$ antibody, the period of infection is debated and may occur at variable times, as reviewed. ${ }^{6,7}$ Nonetheless, association of BD or SZ with infectious agents quite always represent subgroups of patients and may, therefore, play different or additional roles, or may constitute different etiological causes or contributors as suggested by various studies. ${ }^{8-10}$ Genetic studies revealed an overlapped involvement of loci involved in the inflammatory/immune pathways including the major histocompatibility complex region in both $\mathrm{SZ}$ and $\mathrm{BD},{ }^{11-14}$ among other candidate genes. ${ }^{15-17}$ Structural genomic studies also highlighted significant modifications in psychotic patients,

\footnotetext{
${ }^{1}$ Geneuro, Plan-Les-Ouates, Geneva, Switzerland; ${ }^{2}$ Geneuro-Innovation, Pre-Clinical R\&D Department, Lyon, France; ${ }^{3}$ University of Lyon, Ecole Doctorale BMIC, Lyon, France; ${ }^{4}$ Inserm U955, Psychiatrie Génétique, Créteil, France; ${ }^{5}$ AP-HP, Hôpital Henri Mondor-Albert Chenevier, Pôle de Psychiatrie, Créteil, France; ${ }^{6}$ Fondation Fondamental, Créteil, France; ${ }^{7}$ Université Paris Est Créteil, Faculté de Médecine, Créteil, France; ${ }^{8} \mathrm{CEA}$ Saclay, Neurospin, Gif-Sur-Yvette, France; ${ }^{9} \mathrm{AP}$-HP, Université Paris Diderot, Service de Psychiatrie, Hôpital Lariboisiere Fernand Widal, Paris, France; ${ }^{10}$ Jean Dausset Department and INSERM UMRS 940 , Hôpital Saint Louis, Paris, France; ${ }^{11}$ Stanley Laboratory of Developmental Neurovirology, Johns Hopkins University Medical Center, Baltimore, MD, USA; ${ }^{12}$ Plateforme de Ressources Biologiques AP-HP, Créteil, France; ${ }^{13}$ INSERM, UMRS 665, Paris, France; ${ }^{14}$ Stanley Research Program, Sheppard Pratt, Baltimore, MD, USA and ${ }^{15}$ INSERM-CIC 006, Créteil, France

Correspondence: Dr H Perron, Geneuro, 18 Chemin des Aulx, Plan-Les-Ouates, Geneva 1228, Switzerland.

E-mail: hp@geneuro.com

or Professor M Leboyer, AP-HP, Hôpital Henri Mondor-Albert Chenevier, Pôle de Psychiatrie, 40, rue de Mesly, 94010 Créteil, France.

E-mail: marion.leboyer@creteil.inserm.fr

${ }^{16}$ These are senior co-authors.

Keywords: bipolar disorder; copy number variation; HERV-W; MSRV; schizophrenia; toxoplasma

Received 19 September 2012; accepted 21 September 2012
} 
including copy number variations and deletions. ${ }^{18-21}$ Nonetheless, the mechanisms possibly underlying interactions between genetic and environmental risk factors contributing to the clinical onset and/or to the progression of psychotic disorders remain to be understood. ${ }^{16}$

The involvement of atypical genetic elements, such as Human Endogenous Retroviruses (HERVs), has also been reported in SZ and $\mathrm{BD} .^{22-32}$ In schizophrenia (SZ), a sequence homologous to an endogenous retrovirus (ERV) identified in MS and named 'Multiple Sclerosis-associated Retroviral element' (MSRV) ${ }^{33}$ was first identified from differential DNA amplification in homozygote twins discordant for the disease. ${ }^{23}$ MSRV sequences later permitted to unravel a yet unknown HERV family, now named HERV-W. ${ }^{34-37}$ Furthermore, though detected in different brain areas than in MS, many studies have now evidenced white matter and myelin impairment and/or inflammation predominantly in BP but also in SZ. ${ }^{38-44}$ Thus, the expression of the proinflammatory envelope protein of MSRV in MS brain microgliocytes within demyelinating lesions ${ }^{45}$ should now be considered in parallel with myelin alterations in the vicinity of activated microglia in BD and SZ brain.

HERVs have properties of mobile genetic elements causing genetic rearrangements ${ }^{21,46,47}$ potentially influenced by their interactions with microbial agents from environment. ${ }^{48,49}$ Coincidently, important structural modifications in the major histocompatibility complex locus were associated with such characteristics of HERVs. ${ }^{50}$ For these reasons, HERVs may provide a missing link between environmental factors, genetic modifications and pathogeny with downstream neuroinflammation and neurotoxicity in psychotic disorders with such features. ${ }^{21,38,51-54}$

HERVs are components of the genome that can be transmitted to subsequent generations through gametes, but have evolved differently from other host genes. They have significant inter-individual copy number variations within the genome of healthy humans from different ethnic origins ${ }^{55}$ or among subjects with distinct phenotype for example, complement C4 factor. ${ }^{56}$ These multicopy families have retained characteristics of retroviruses. ${ }^{21}$ In fact, HERVs, which represent $8 \%$ of the human genome, ${ }^{57}$ are part of the superfamily of repeated and transposable elements (transposons, retrotransposons and ERVs) representing about $42 \%$ of the human genome. ${ }^{58}$ They probably had a role in interindividual gene transmission, as well as in intracellular gene retrotransposition or recombination, and may undergo changes under selective environmental pressure. ${ }^{21,59}$ In certain conditions undisrupted HERV sequences may be expressed and display viral protein properties. ${ }^{49}$ Although most of the contemporary copies of HERVs are inactivated by mutations or deletions or silenced by epigenetic modifications, $^{60}$ their plasticity and potential responsiveness to environmental triggers are of particular relevance for gene-environment interactions. ${ }^{21}$ In the case of the HERVW family and its MSRV element, it has now consistently been shown that certain infectious agents can trigger activation of certain copies. ${ }^{61}$ In particular, HERV-W elements have been reported to be activated by $T$. gondii, ${ }^{62}$ as well as by Influenza virus $^{48}$ in human cell lines. Such pathogenic activation of HERV-W, mainly focusing on MSRV-type elements in experimental or clinical studies, ${ }^{45,63-65}$ may result in the production of its envelope protein (HERV-W Env) that strongly stimulates a pro-inflammatory cascade through the TLR4 receptor pathway ${ }^{66}$ and displays potential neurotoxicity. ${ }^{25}$ We, therefore, considered that these highly relevant features place HERV-W elements at the forefront of geneenvironment interactions underlying complex diseases such as SZ. ${ }^{49,51}$

In the present study, we have further investigated the genetic features and the ex-vivo transcriptional activity of HERV-W envelope copies, as reflected in appropriate blood cells, in patients with $\mathrm{SZ}$ and $\mathrm{BD}$ in comparison with healthy controls (HC). Moreover, as MSRV has now specifically been shown to have detectable and abnormal expression in the peripheral mononuclear cells (PBMC, representing of lymphocytes and monocytes) of patients with $M S,{ }^{45,67}$ the same technical approach was applied here. The cellular RNA and genomic DNA copies were thus quantified in PBMC from patients with $\mathrm{BD}$, with $\mathrm{SZ}$ and from $\mathrm{HC}$, using an established real-time PCR technique targeting the MSRV subtype of HERV-W family. ${ }^{67}$

\section{Patients and methods}

Participants. Patients fulfilling DSM-IV criteria (American Psychiatric Association, 1994) for SZ or BD were recruited during hospitalization or follow-up visits in two universityaffiliated psychiatric departments (Paris, France). Inclusion criteria for study participation were: age between 18 and 65 years, no history of alcohol or drug abuse/dependence, no history of mental retardation, no previous head trauma with loss of conscience. Patients were interviewed with the French version of the 'Diagnostic Interview for Genetic Studies'. ${ }^{68}$ Patients with SZ were evaluated with the Positive And Negative Syndrome Scale ${ }^{69}$ and with the Calgary scale ${ }^{70}$ measuring the depressive symptoms. Patients with BD were screened with the Young Mania Rating Scale ${ }^{71}$ searching for manic symptoms, and with the Montgomery and Asberg Depression Rating Scale ${ }^{72}$ for depressive symptoms.

$\mathrm{HC}$ without any personal and family history of SZ or BD were enrolled through a clinical investigation center. Patients and controls had negative serology for human immunodeficiency viruses $(H I V 1+2)$, Hepatitis $A, B$ and $C$ viruses, no known inflammatory, auto-immune or neurological disorder. All subjects gave written informed consent for their participation in this study with ethical committee approval.

Serum collection. One tube $(7 \mathrm{ml}$ dry tube B\&D, Meylan, France) of blood from each subject was treated within $2 \mathrm{~h}$ after collection. The clotted blood was centrifuged for $10 \mathrm{~min}$ at $2800 \mathrm{~g}$ at $+4{ }^{\circ} \mathrm{C}$. Clear serum (hemolytic sera were rejected) was collected and stored at $-20^{\circ} \mathrm{C}$.

Serological analyses. Immunoglobulin $G$ antibodies were measured as previously described ${ }^{73}$ in the Stanley Division of Developmental Neurovirology (Baltimore, USA). (1) For viruses of the Herpesvirus family: HSV-1, HSV-2, CMV, EBV, HHV-6 and VZV, (2) for T. gondii. Assays for HSV-1 and HSV-2 used envelope glycoproteins gG1 and gG2, respectively. 
Quantitative HERV-W PCR. PBMCs were tested according to a previously described quantitative PCR (qPCR) and reverse transcriptase PCR (RT-PCR) specific for MSRV subtype of HERV-W family ${ }^{67}$ further adapted as detailed.

Quantification of MSRV-env RNA in PBMC by real-time RT-PCR. All steps, from the extraction of RNA to the interpretation of the final results, were conducted in blinded conditions and according to a standard operation procedure (SOP, Geneuro-Innovation, Lyon, France). Briefly, PBMCs were washed with phosphate-buffered saline and total RNA was extracted with RNeasy Mini Kit (Qiagen, Hilden, Germany) according to the manufacturer's instructions. RNA was treated with Turbo DNA-free kit (Applied Biosystems, Foster City, CA, USA) before assessment of its quality and concentration by spectrophotometer; then sample volumes were adjusted to add $50 \mathrm{ng}$ of RNA per reaction. For each sample, the expression (calculated as the cycling threshold or $\mathrm{Ct}$ ) of MSRV-env and the housekeeping gene GUS $B$ encoding the glucuronidase beta was analyzed in triplicate by RT-PCR (Thermal Cycler C1000_CFX96 RealTime System, Bio-Rad).

Specific sets of primers and probes for MSRV-env (according to Mameli et al. ${ }^{67}$ ) and GUS $B$ (Taqman gene expression assay -GUS B, ref. 4331182/Hs99999908_m1, Applied Biosystems) were used to perform a One-Step RT-PCR (iScript One-Step RT-PCR for Probes, Bio-Rad) with an initial step of $50^{\circ} \mathrm{C}$ for $10 \mathrm{~min}$ and $95^{\circ} \mathrm{C}$ for $5 \mathrm{~min}$ incubations followed by 45 cycles of $95^{\circ} \mathrm{C}$ for $10 \mathrm{~s}$ and $60^{\circ} \mathrm{C}$ for $1 \mathrm{~min}$.

For each sample a control without reverse transcriptase was analyzed to detect possible DNA contamination. Assays were considered acceptable if: (a) amplification of the housekeeping gene was detected in all the extracted samples; (b) no amplification was detected without reverse transcriptase; (c) the efficiency of the PCR was 90-110\% (3.6> slope > 3.1); (d) variability among replicates was $<5 \%$.

Results, representing standardized MSRV RNA expression levels, are expressed as MSRV-env relative expression to the reference and stably expressed GUS B RNA for each subject,

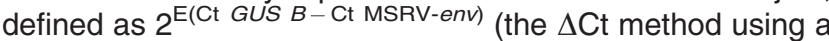
reference gene, Real-Time PCR Application Guide, Bio-Rad Laboratories).

The normal copy number of transcript reflecting the background transcriptional activity of various HERV-W copies, mostly defective as described for ERVs, ${ }^{21}$ is determined from the $\mathrm{HC}$.

A threshold (cutoff value of the technique) above which an elevated transcriptional activity of HERV-W becomes significant, was, therefore, determined from the mean of the $\mathrm{HC}$ population plus twice its s.d. $(M+2$ s.d.). Outliers corresponding to $\mathrm{HC}$ individuals with values above the mean plus its s.d. $(\mathrm{M}+\mathrm{s} . \mathrm{d}$.) are not included in the calculation of this 'cutoff' value.

MSRV-env genomic DNA copy number in total blood leukocytes by real-time PCR. DNA was extracted from peripheral blood leukocytes using a salting out procedure. DNA concentration and quality were assessed using Qubit ${ }^{\mathbb{R}}$ double stranded DNA BR Assay Kits and Qubit ${ }^{\mathbb{R}} 2.0$
Fluorometer (Life Technologies, Carlsbad, CA, USA) and volumes were adjusted to add $20 \mathrm{ng}$ of DNA per reaction.

Relative DNA quantification was performed using the same MSRV primers ${ }^{67}$ in parallel with a reference standard consisting in a monocopy human gene encoding the hydroxymethylbilane synthase.

PCR reactions were performed in a final volume of $20 \mu \mathrm{l}$, containing $20 \mathrm{ng}$ of DNA, $0.5 \mu \mathrm{m}$ of forward and reverse primers, $0.2 \mu \mathrm{m}$ of fluorescently labeled specific probe and $1 \times$ of TaqMan Universal Mastermix (Applied Biosystems), and run in a Mastercycler ${ }^{\circledR}$ ep realplex2S (Eppendorf, Hamburg, Germany). PCR cycle parameters were $50{ }^{\circ} \mathrm{C}$ for $2 \mathrm{~min}, 95^{\circ} \mathrm{C}$ for $10 \mathrm{~min}, 50$ cycles of $95^{\circ} \mathrm{C}$ for $15 \mathrm{~s}$ and $60^{\circ} \mathrm{C}$ for $1 \mathrm{~min}$. Common threshold fluorescence for all the samples was set into the exponential phase of the amplification and determined the $\mathrm{Ct}$, corresponding to the number of amplification cycles needed to reach this threshold. All reactions were performed in triplicate and the mean value of $\mathrm{Ct}$ was used for subsequent analysis.

Results, representing the MSRV DNA copy number per diploid human genome, were obtained by calculating the ratio between HERV-W/MSRV copies and hydroxymethylbilane synthase copies in the same sample.

When samples did not allow RNA and/or DNA extraction in sufficient quantity or quality, they were not tested and corresponding patients not analyzed in the test series.

PCR product sequencing. MSRV-Env DNA and RNA amplicons were cloned according to the manufacturer's instructions for the TOPO-cloning kit (TOPO ${ }^{\circledR}$ TA Cloning ${ }^{\circledR}$ Kit, Invitrogen, Carlsbad, New-Mexico, USA) followed by capillary sequencing (Applied Biosystems ${ }^{\mathbb{R}}$ ), by Fasteris SA Plan-Les-Ouates (Switzerland). Sequences were analyzed using Mac Vector ${ }^{\mathbb{B}}$ v11.1.1software (MacVector Inc., Cambridge, NC, USA). Multiple sequence alignments were performed using clustalW (v1.83) multiple alignment algorithm. Phylogenic analysis was performed using a neighbor joining bootstrap with 1000 repeats and Kimura 2-parameters method for graphical 'tree' presentation.

Statistical analysis. Figures from BD, SZ and HCs groups were compared using $\chi^{2}$-test for categorical data and the Kruskal-Wallis or Mann-Whitney test for nonparametric data

All statistical analyses were performed using $\mathrm{R}$ software package version 2.13.0 (Stanford University Social Science Data and Software, Stanford, CA, USA).

\section{Results}

Demographical characteristics. The study group consisted of 136 patients ( 91 with BD and 45 with SZ) and 73 HC. Half of them (52.2\%) were men (47.3\% for BD, $75.6 \%$ for $\mathrm{SZ}$ and $43.8 \%$ for $\mathrm{HC}$ ) with a mean age at interview of 38.02 $( \pm 12.7)$ years. Patients with BD were significantly older than patients with $\mathrm{SZ}$ and HCs $(43.81 \pm 12.4,34.75 \pm 11.1$ and $37.33 \pm 12.8$ years, respectively, $P<=0.0001)$.

For patients with $\mathrm{BD}$, the mean scores of depression (measured with the Montgomery and Asberg Depression 
Rating Scale) and of mania (measured with Young Mania Rating Scale) were $6.54 \pm 8.9$ and $4.92 \pm 7.4$, respectively. In the sample of patients with SZ, mean scores of depression (measured with the Calgary) and of psychotic symptoms (measured with the Positive And Negative Syndrome Scale) were of $8.45 \pm 6.7$ and $65.46 \pm 17.5$, respectively. Thus, patients with BD were mostly euthymic, and patients with SZ were not in an acute phase. The majority of them were patients with long-duration disorder: mean of $16.91 \pm 11.2$ years for BD and $10.93 \pm 9.1$ years for SZ.

The majority of patient with $\mathrm{BD}$ received a mood stabilizer (lithium in $35.4 \%$, valproate in $20.9 \%$, atypical antipsychotics in $4.1 \%$, a combination of two mood stabilizers in $12.5 \%$, a combination of a mood stabilizer and an atypical antipsychotic in $21.9 \%$ of the cases). Patients with SZ received atypical antipsychotics in $70.4 \%$ of the cases, typical antipsychotics in $7.4 \%$ and a combination of a mood stabilizer and an atypical antipsychotic in $22.2 \%$ of the cases.

HERV-W RNA expression. As presented in Figure 1a, the levels of HERV-W RNA transcription determined by qPCR were significantly elevated in PBMC of patients with $B D$ and patients with $\mathrm{SZ}$, compared with $\mathrm{HC}\left(P<10^{-4}\right.$ for $\mathrm{BD}$ and $P=0.012$ for SZ). When comparing the two groups of patients, BD group had a significantly higher expression than SZ group $(P<0.01)$. When patients with SZ or BD were compared with $\mathrm{HC}$ with a C-reactive protein below the normal threshold (C-reactive protein $<5$; ' $\mathrm{C}$-' subgroup), the statistical significance remained unchanged for all comparisons, or even increased for SZ versus C- $(P=0.007)$.

In Figure 1b, the distribution of HERV-W RNA levels is presented for all clinical categories. A different distribution was observed between patients and controls, with the expected distribution in $\mathrm{HC}$ peaking at the lowest value.

Patients were further stratified according to the transcriptional levels of HERV-W RNA in PBMC: patients with values above the cutoff (mean +2 s.d. of $\mathrm{HC}$; see Patients and methods) were considered to have significantly elevated values (positives), whereas patients with values below this threshold were considered to have normal RNA levels (negatives).

As presented in Table 1a, we found no correlation between HERV-W RNA 'positives' or 'negatives', with respect to age at onset of the disorder, duration of illness, number of prior
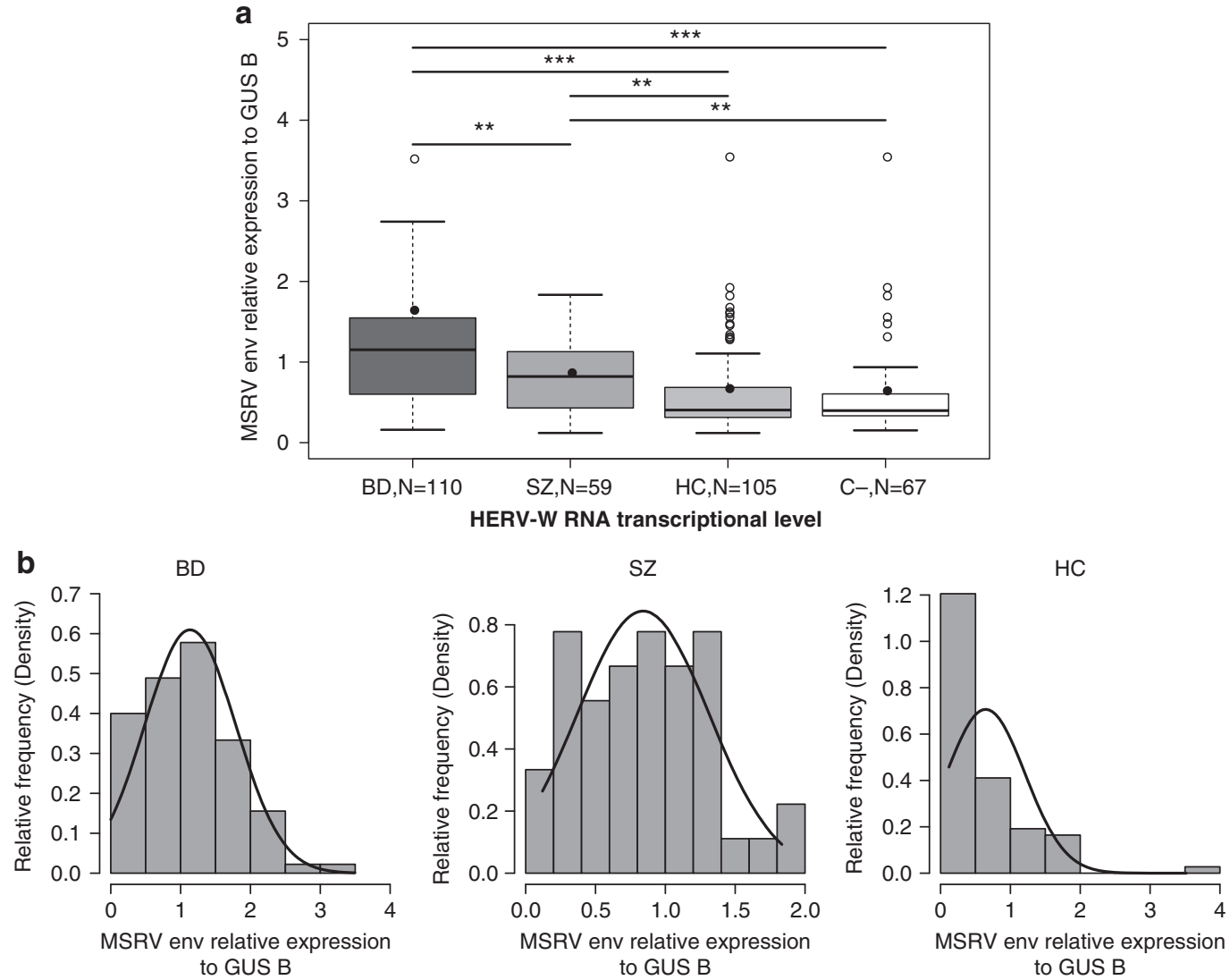

Figure 1 Human Endogenous Retrovirus type-W (HERV-W) RNA transcriptional level. (a) HERV-W env RNA expression in patients and control groups. The relative transcription of Multiple Sclerosis-associated Retroviral element (MSRV)-env RNA to the housekeeping gene GUS B is indicated on the y axis; it measures the standardized

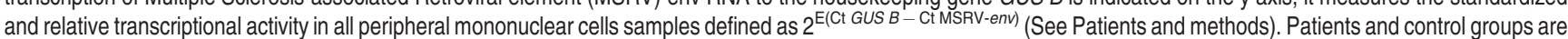
indicated on the $\mathrm{x}$ axis: $\mathrm{BD}$, bipolar disorder; SZ, schizophrenia; HC, healthy controls without psychiatric history; C-, subset of $\mathrm{HC}$ group with C-reactive protein below the normal threshold in serum. ${ }^{* \star} P \leqslant 0.01{ }^{* \star *} P \leqslant 0.0001$. (b) Distribution of HERV-W RNA expression levels in patients and control groups. y axis represents the density of corresponding HERV-W env RNA relative expression values on the $x$ axis, giving a description of their distribution in each group (BD, SZ, HC). Density curve: the area under a density curve (between the curve and the horizontal axis) is always defined as 1 unit. The area under the density curve between two values is the proportion of observations in the data set that fall between those two values. Histogram: the distribution frequency of results between two values is represented by bars. 
Table 1a Clinical and serological status according to HERV-W RNA transcriptional level (HERV + and HERV - )

\begin{tabular}{|c|c|c|c|c|c|c|c|c|}
\hline & \multicolumn{4}{|c|}{$B D$} & \multicolumn{4}{|c|}{$S Z$} \\
\hline & $\begin{array}{c}\mathrm{N} \text { used } \\
( \pm)\end{array}$ & $\begin{array}{l}H E R V+ \\
(\mathrm{N}=51)\end{array}$ & $\begin{array}{l}H E R V- \\
(\mathrm{N}=40)\end{array}$ & $\begin{array}{l}\mathrm{P}- \\
\text { value }\end{array}$ & $\begin{array}{c}\mathrm{N} \text { used } \\
( \pm)\end{array}$ & $\begin{array}{l}H E R V+ \\
(\mathrm{N}=17)\end{array}$ & $H E R V-(\mathrm{N}=28)$ & $\begin{array}{l}\mathrm{P}- \\
\text { value }\end{array}$ \\
\hline Age at onset (years \pm s.d. ) & $48 / 39$ & $25.83(8.6)$ & $27.28(11.6)$ & 0.931 & $16 / 26$ & $24.06(10)$ & $23.65(5.8)$ & 0.773 \\
\hline Age at interview (years \pm s.d.) & $49 / 40$ & $44.55(12)$ & $42.9(13.2)$ & 0.723 & $16 / 25$ & $33.93(11.1)$ & $35.28(11.3)$ & 0.749 \\
\hline $\begin{array}{l}\text { Duration of illness } \\
\text { (years } \pm \text { s.d.) }\end{array}$ & $49 / 39$ & $18.30(11.9)$ & $15.17(10.1)$ & 0.220 & $16 / 25$ & $9.88(6.4)$ & $11.6(10.5)$ & 0.952 \\
\hline Number of episodes & $43 / 39$ & $8.62(6.3)$ & $8.33(7.8)$ & 0.270 & $15 / 24$ & $4.10(3.1)$ & $4.12(3)$ & 0.898 \\
\hline MADRS (mean scores) & $49 / 40$ & $6.18(9.1)$ & $6.98(8.9)$ & 0.792 & & & & \\
\hline YMRS (mean scores) & $49 / 39$ & $4.50(7.1)$ & $5.43(7.8)$ & 0.727 & & & & \\
\hline PANSS (mean total scores) & $45 / 35$ & $37.13(11.5)$ & $38.94(13.3)$ & 0.896 & $15 / 20$ & $65.93(20.9)$ & $65.1(15.1)$ & 0.469 \\
\hline
\end{tabular}

Abbreviations: BD, (patients with) bipolar disorder; HERV-W, Human Endogenous Retrovirus type-W; MADRS, Montgomery and Asberg Depression Rating Scale; PANSS, Positive And Negative Syndrome Scale; SZ, (patients with) schizophrenia;YMRS, Young Mania Rating Scale.

HERV-W expression status among patients with BD and SZ.

HERV-W 'Positives' have a transcriptional level with RNA copy number significantly elevated, compared with background transcription in healthy controls; 'Negatives' have RNA copy number below the threshold (see Patients and methods).

As few samples could not be tested for all parameters (insufficient volume, quality control not passed, and so on) the numbers effectively tested and used for calculations presented in Table 1 a are indicated in columns with ' $N$ used $( \pm)$ '.

Table 1b Clinical and serological status according to HERV-W RNA transcriptional level (HERV + and HERV - )

\begin{tabular}{|c|c|c|c|c|c|c|c|c|c|}
\hline \multirow{3}{*}{$\begin{array}{l}\text { Variables, number of tested sera ( } N \text { used: } \\
\text { HERV }+/ \text { HERV }- \text { ) }\end{array}$} & \multicolumn{3}{|c|}{ All patients, $S Z$ and $B D$} & \multicolumn{3}{|c|}{$B D$} & \multicolumn{3}{|c|}{$S Z$} \\
\hline & $\begin{array}{l}H E R V+ \\
\quad(68)\end{array}$ & $\begin{array}{c}H E R V- \\
\quad(68)\end{array}$ & $\mathrm{P}$-value & $\begin{array}{c}H E R V+ \\
\quad(51)\end{array}$ & $\begin{array}{l}H E R V+ \\
\quad(40)\end{array}$ & P-value & $\begin{array}{c}H E R V+ \\
\quad(17)\end{array}$ & $\begin{array}{l}\text { HERV - } \\
\text { (28) }\end{array}$ & P-value \\
\hline & \multicolumn{2}{|c|}{$\begin{array}{c}\text { Positive serology } \\
\text { (\% pos) }\end{array}$} & & \multicolumn{2}{|c|}{$\begin{array}{c}\text { Positive serology } \\
\text { (\% pos) }\end{array}$} & \multicolumn{4}{|c|}{$\begin{array}{c}\text { Positive serology } \\
\text { (\% pos) }\end{array}$} \\
\hline $\begin{array}{l}\text { T. Gondii, (63/56) } \\
\text { HSV-1, (63/56) } \\
\text { HSV-2, (63/56) } \\
\text { CMV, }(63 / 56)\end{array}$ & $\begin{array}{l}53(84.1 \%) \\
41(65.1 \%) \\
14(22.2 \%) \\
36(57.1 \%)\end{array}$ & $\begin{array}{l}38(67.9) \\
31(55.4) \\
12(21.4) \\
25(44.6)\end{array}$ & $\begin{array}{l}0.036 \\
0.279 \\
0.917 \\
0.173\end{array}$ & $\begin{array}{l}42(84) \\
33(66) \\
14(28) \\
30(60)\end{array}$ & $\begin{array}{l}25(69.4) \\
22(61.1) \\
10(27.8) \\
17(47.2)\end{array}$ & $\begin{array}{l}0.109 \\
0.641 \\
0.982 \\
0.24\end{array}$ & $\begin{array}{l}11(84.6) \\
8(61.5) \\
0(0) \\
6(46.2)\end{array}$ & $\begin{array}{r}13(65) \\
9(45) \\
2(10) \\
8(40)\end{array}$ & $\begin{array}{l}0.216 \\
0.353 \\
0.239 \\
0.727\end{array}$ \\
\hline
\end{tabular}

Abbreviations: BD, (patients with) bipolar disorder; CMV, Cytomegalovirus; HERV-W, Human Endogenous Retrovirus type-W; HSV-1 or -2, Herpes Simplex Virus Type 1 or 2; SZ, (patients with) schizophrenia.

HERV-W expression status among patients with BD and SZ.

HERV-W 'Positives' have a transcriptional level with RNA copy number significantly elevated, compared with background transcription in healthy controls; 'Negatives' have RNA copy number below the threshold (see Patients and methods).

As few samples could not be tested for all parameters (insufficient volume, quality control not passed, and so on), the numbers effectively tested and used for calculations presented in Table $1 \mathrm{~b}$ are indicated in columns with ' $N$ used $( \pm)$ ".

episodes, frequency of hospitalizations, Positive and Negative Syndrome Scale scores for patients with SZ and Young Mania Rating Scale or Montgomery and Asberg Depression Rating Scale scores for BD. As valproic acid (valproate) was found to be associated with elevated HERV transcription in a previous study, ${ }^{74}$ we compared patients with BD treated or not with valproic acid. We observed that patients with $B D$ treated with valproic acid had higher RNA levels $(P=0.035)$ than valproate-free patients. Therefore, we excluded all patients treated with valproic acid and analyzed valproate-free subgroups of patients with BD and SZ. A significant difference was still observed between $\mathrm{HC}$ and $\mathrm{BD}$ or patients with $\mathrm{SZ}$ ( $P<0.0001$ and $P=0.007$, respectively).

The BD and SZ groups, in which a great majority of patients presented chronic and/or stable disease as above described, showed no significant correlation with the other tested clinical parameters (Table 1a).

Further, patients with BD or SZ did not differ from controls for seropositivity (immunoglobulin $\mathrm{G}$ antibodies) to $T$. gondii,
HSV 1and 2, and for CMV. Similarly, SZ did not differ from BD. Nevertheless, the seroprevalence of $T$. gondii was significantly higher in patients with $\mathrm{BD}$ and $\mathrm{SZ}$, when merged as a single group, than in $\mathrm{HC}(P<0.036$; Table $1 \mathrm{~b})$.

HERV-W DNA genomic copy number. As presented in Figure 2, HERV-W MSRV-type DNA copy number was significantly lower in DNA of leukocytes from patients with $B D$ and $\mathrm{SZ}$ as compared with $\mathrm{HC}(P=0.0016$ and $P<0.0003$ for BD and SZ, respectively; Supplementary Information). However, no significant difference in the genomic HERV-W DNA copy number was found between BD and SZ, nor between $\mathrm{HC}$ and $\mathrm{C}$ - (C-reactive protein-negative controls; $p>0.5)$. In Figure $2 b$, the distribution profile of HERV-W DNA copy number is presented for all categories, with figures of $\mathrm{HC}$ peaking at the center (normal distribution). Even after excluding patients treated with valproic acid, the difference remained unchanged and statistically significant (Supplementary Information). 

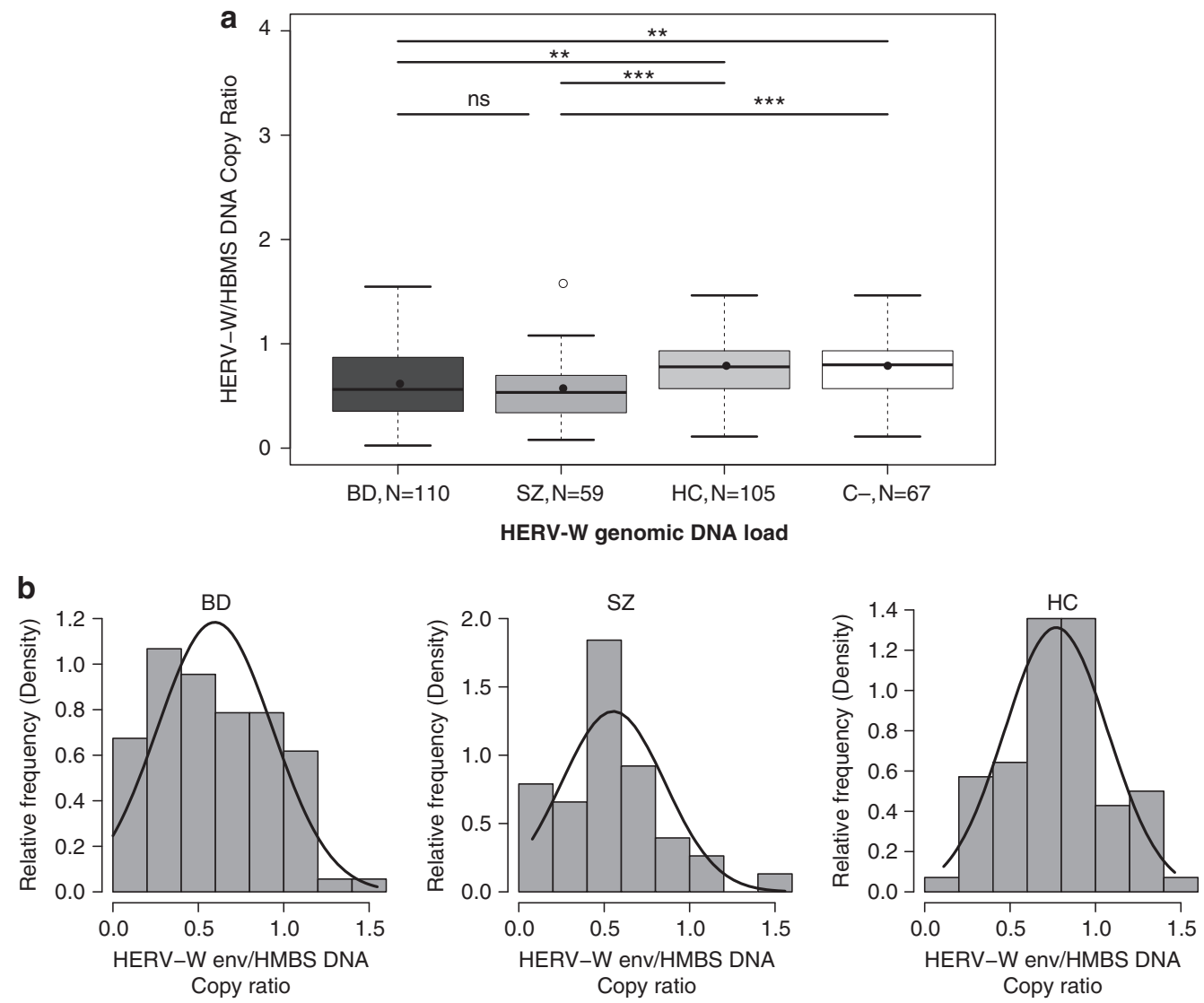

Figure 2 Human Endogenous Retrovirus type-W (HERV-W) genomic DNA load. (a) Relative HERV-W DNA copy number in patients and controls. The ratio between the DNA copy number of HERV-W env gene Multiple Sclerosis-associated Retroviral element (MSRV)-subtype and the hydroxymethylbilane synthase housekeeping gene is indicated on the $y$ axis. This measures the relative quantification of copy numbers from the MSRV subtype, detected by the MSRV-env probe with the quantitative PCR technical conditions used here on total blood leukocytes. Patients and control groups are indicated on the x axis: BD, bipolar disorder; SZ, schizophrenia; HC, healthy controls without psychiatric history; $\mathrm{C}$-, subset of $\mathrm{HC}$ group without $\mathrm{C}$-reactive protein above the normal threshold in serum. ${ }^{* *} P \leqslant 0.01{ }^{* * *} P \leqslant 0.0001$. (b) Distribution of HERV-W env DNA copy number in patients and controls. y Axis represents the frequency and the density of corresponding HERV-W env DNA copy values on the $x$ axis, giving a description of their distribution in each group (BD, SZ, HC). Density curve: the area under a density curve (between the curve and the horizontal axis) is always defined as 1 unit. The area under the density curve between two values is the proportion of observations in the data set that fall between those two values. Histogram: the distribution frequency of results between two values is represented by bars.

Sequence analyses of HERV-W PCR amplicons. The PCR products were cloned and sequenced to address the specificity of the qPCR technique, as well as eventual qualitative difference.

The PCR products from randomly selected individuals (three $\mathrm{HC}$, three patients with BD and four with SZ; Table 2) provided a representative panel of DNA and RNA amplicons from each category. In order to avoid mistakes in reassembling irrelevant fragments with overlapping sequences by deep sequencing of short nucleotide stretches within a complex mixture of variants, PCR products were cloned. Inserts were sequenced on both strands and then aligned with the probe used for qPCR. Sequences showing significant alignment, here with a maximum of two mismatches (see Patients and methods), correspond to the amplicons that determine the copy number measured by this probe in $\mathrm{qPCR}$.

Table 2 shows that the highest percentage of clones identical to the reference probe was obtained for RNA from SZ $(87 \%, 33 / 38)$. Conversely, in RNA from BD, the majority of clones had one or two nucleotide mismatches $(74 \%, 26 / 35)$. Finally, in RNA from $\mathrm{HC}$, all sequences presenting mismatches (12/34) were absent in RNA of either patients with SZ $(0 / 38)$ or $\mathrm{BD}(0 / 47)$. This difference was significant when compared with either BD or SZ group $\left(\chi^{2}: P<0.001\right)$.

Corresponding sequence alignments are shown in Supplementary Information.

Detailed analysis was performed with HERV-W reference clones (MSRV-env, ERVWE1 encoding Syncytin on chromosome 7) and a distant envelope gene from another HERV family (HERV-K113-env) followed by phylogenic analysis (Supplementary Information and Patients and methods). Significant homology with HERV-W env sequence obtained from retroviral RNA within purified extracellular virions ${ }^{33,75}$ was found for most sequences, which emphasizes the optimized selectivity of the present PCR conditions for this subtype of HERV-W family and explains the limited number of copies detected within human DNA.

These qualitative differences in the frequency of variants among $\mathrm{BD}, \mathrm{SZ}$ and $\mathrm{HC}$ are additional discriminating features 
Table 2 HERV-W env clones obtained from PBMC by standard PCR with primers used in the quantitative PCR

\begin{tabular}{|c|c|c|c|c|}
\hline \multirow[t]{2}{*}{ Status } & DNA PCR & $D N A P C R$ & $R N A R T-P C R$ & $R N A R T-P C R$ \\
\hline & $\begin{array}{l}\text { Number of } \\
\text { clones with } \\
\text { insert }\end{array}$ & $\begin{array}{c}\text { Number of } \\
\text { sequences } \\
\text { aligned with } \\
\text { probe }\end{array}$ & $\begin{array}{l}\text { Number of } \\
\text { clones with } \\
\text { insert }\end{array}$ & $\begin{array}{c}\text { Number of } \\
\text { sequences } \\
\text { aligned with } \\
\text { probe }\end{array}$ \\
\hline $\begin{array}{l}S Z \\
B D \\
H C\end{array}$ & $\begin{array}{l}32 \\
36 \\
36\end{array}$ & $\begin{array}{l}3(0) ; 3(1) ; 19(2) \\
5(0) ; 5(1) ; 18^{\mathrm{b}}(2) \\
10(0) ; 8(1) ; 16(2)\end{array}$ & $\begin{array}{l}38 \\
47 \\
34\end{array}$ & $\begin{array}{c}33(0) ; 4(1) ; 0(2) \\
9(0) ; 20(1) ; 6(2) \\
20(0) ; 11^{\mathrm{a}}(1) ; 1^{\mathrm{a}}(2)\end{array}$ \\
\hline
\end{tabular}

Abbreviations: BD, bipolar disorder; HC, healthy controls; HERV-W, Human Endogenous Retrovirus type-W; PBMC, peripheral mononuclear cell; RT-PCR, reverse transcriptase PCR; SZ, schizophrenia.

Clones were obtained in PBMC from three $\mathrm{HC}$, three $\mathrm{BD}$ and four $\mathrm{SZ}$ individuals. According to software analyses (Mac Vector V.11.1.1, Cambridge, NC, USA) amplicons with a maximum of two nucleotide substitutions could hybridize with the probe used in the quantitative PCR assay. The number of clones are indicated followed by the corresponding number of substituted nucleotides between brackets for 0,1 and 2 possible substitutions.

Detailed sequences with alignments are presented in Supplementary Information.

aNucleotide substitutions never found in RNA from SZ, but in 19 clones from BD RNA. This sequence is nonetheless present in DNA: one clone from SZ, three clones from $\mathrm{BD}$ and two clones from HC. ${ }^{\mathrm{b}} \mathrm{Clones}$ with two nucleotide substitutions from BD DNA are all identical, but never found in clones from RNA (BD, SZ or HC ). They are present in 14/19 clones from SZ DNA and 14/16 clones from HC DNA.

to the observed differences in RNA and DNA copy numbers (Figures $1 \mathrm{a}$ and $2 \mathrm{a}$ ) and in their distribution (Figures $1 \mathrm{~b}$ and $2 b)$.

\section{Discussion}

The present molecular study is consistent with previously reported association between HERV-W expression and $S Z,{ }^{6,51}$ but also unravels the existence and specificities of an analogous association between BD and HERV-W env.

Moreover, transcriptional levels of HERV-W env RNA from patients with BD here appear significantly higher than among patients with SZ, with additional qualitative differences in nucleotide sequences. This is obviously not attributable to treatment as most patients were treated and no correlation between a given treatment and results was evidenced. In particular, valproic acid had no impact as previously suggested, ${ }^{74}$ as excluding patients treated with this mood stabilizer yielded similarly significant differences with $\mathrm{HC}$. It therefore cannot account for the quantitative and qualitative molecular findings in patients with BD or with SZ.

The significance of an elevated HERV-W env RNA expression in SZ and BD groups was also confirmed by comparison with a subgroup (C-) excluding controls with elevated C-reactive protein, a nonspecific marker of inflammation ${ }^{76}$ indicative of risk for various diseases in apparently healthy individuals. ${ }^{77}$

The influence of disease state could also be debated as previous studies on RNA only included patients with SZ during first or acute psychotic episode, or patients with BD during acute mood episode. For the first time, our present populations of patients with BD, who were mostly euthymic, and of patients with SZ, most of whom were not in an acute phase, evidence that this HERV-W expression is not simply linked to, or a mere consequence of, active episodes of these diseases.
Importantly, it can now be assumed that elevated HERV-W/ MSRV RNA expression appears constitutive in these patients.

Nonetheless, this does not preclude more elevated RNA expression that might peak during active and highly symptomatic periods of patients with BD or SZ. Moreover, only a subset of patients (with first onset or symptomatically active) have been shown to be positive for HERV-W env mRNA expression in independent studies, ${ }^{22,24,25,50}$ which is also the case with the present study on stable patients $(62 \%-28 / 45$ of positives for SZ; $56 \%-51 / 91$ of positives for BD). Therefore, eventual transiently 'negative' RNA expression cannot be excluded during the clinical course of these illnesses. Alternatively, this association with HERV-W may only be relevant in a subgroup of patients with $S Z$ and BD, which would point to different pathogenic associations in 'HERV-W negative' groups. BD and SZ disease entities are only defined by clinical criteria and might comprise subgroups with different etiopathogenic factors, but future longitudinal follow-up studies are now needed to address such important questions.

The unexpected low DNA copy number of HERV-W env in genomes of patients with SZ or BD (as compared with $\mathrm{HC}$ ) is also accompanied by qualitative differences both in the distribution of copy numbers and in the nucleotide sequences within the probe region. In addition, despite similar DNA copy numbers, patients with SZ or BD show differing distributions of RNA transcription levels and of nucleotide sequences.

Altogether, these data confirm a consistent association with SZ and BD of this MSRV-type of HERV-W family that was first isolated in $\mathrm{MS}^{33}$ However, the patterns of association between MS or SZ/BD appear quite different for the DNA, as well as for nucleotide sequence features of both RNA and DNA.

Results of the present study could, therefore, suggest that differences in HERV-W panel (number and nature of DNA copies) may preexist in the genome of newborns diagnosed later in their life with SZ or BD. An atypical transcriptional potency could result from inherited HERV-W variants with low copy number. Alternatively, de novo acquired modifications of such 'mobile genetic elements' cannot be totally excluded, as reported in other conditions. ${ }^{21}$

In animals, analogous situations are encountered with families of Endogenous Retroviruses (ERV), in which pathogenic ERV copies are unevenly distributed in species genomes. The presence of particular and/or numerous ERV 'defective' copies can be protective against pathogenic ERV strains, while their absence may be detrimental. ${ }^{16,51,52}$ The hypothesis of de novo acquisition of genetic alterations could also be consistent with numerous studies associating intrauterine/perinatal infections or inflammatory events as potential triggers in the development of $\mathrm{BD}$ or $\mathrm{SZ}$ later in life. ${ }^{51}$ Genetic modifications could affect the HERV-W copy numbers as insertion/deletion phenomena have been reported in association with HERVs, ${ }^{56}$ but could also affect the detection of modified HERV-W copies by the present PCR primers and probe. This alternative explanation may thus shed light on the well-described genetic rearrangements in SZ or BD, ${ }^{16}$ with small to large deletions and/or copy number variations in the genome of affected individuals, as HERV rearrangements are also known to involve neighboring genes. ${ }^{56}$ 
Although the mechanisms are not yet elucidated, activation of HERV-W elements epigenetically silenced in differentiated cells was put forward. ${ }^{49}$ Here, the only environmental agent showing some degree of association with enhanced HERV-W RNA expression is $T$. gondii. It nonetheless required large numbers with the merged SZ and $B D$ groups to reach statistical significance and appeared relevant in a subgroup of patients. The fact that this parasite often had increased prevalence in cohorts of patients with $\mathrm{BD}$ or $\mathrm{SZ}^{78}$ would then be consistent with a role as a pathogenic co-factor, which can be replaced by other co-factors displaying analogous effects. Alternatively, it might have a role in association with particular symptomatology, as suggested by a recent study. ${ }^{9}$ Interestingly, T. gondii is known to transactivate HERVs, including HERV-W elements, when infecting tumor cells, ${ }^{62,79}$ which would also fit with a co-factor triggering HERV-W activation with subsequent genetic and inflammatory effects related to HERV-W envelope. This points to an indirect role of such infectious agents through 'epigenetically susceptible' HERV elements, as it is known that tumor cells have extensive DNA hypomethylation, ${ }^{80,81}$ which occurs physiologically in embryonic cells. ${ }^{82}$ Thus, T. gondii could induce a targeted activation of HERV-W elements creating a risk for SZ or BD in individuals carrying an HERV-W 'pathogenic element'. Such HERV-W elements appear here to fit with the MSRV subtype detected under the form of circulating retrovirus-like particles in a low proportion $(8.9 \pm 6.2 \%)$ of studied Caucasian populations. ${ }^{83}$ Thus, requiring the coincidence of both triggering and responding entities for the genesis of HERV-W pathogenicity, environmental pathogens other than T. gondii may not yield significant results with our present numbers and patient selection, due to their variability as co-factors and to their less frequent prevalence in our cohorts.

We thus hypothesize that, at a particular vulnerable developmental stage (for example, when the panel of HERV-W genes is hypomethylated and/or with low epigenetic control), an environmental trigger such as an infection favors cell lineage-specific genetic modifications in these elements (retrotransposition, gene rearrangements, and so on) establishing altered/variable patterns of neurodevelopment. Later in life, each of these patterns would respond differently to subsequent environmental triggers and be translated into distinct clinical phenotypes such as SZ, BD. Quite different nature and conditions of interactions with HERV-W elements (for example, no association ever found with $T$. gondii nor with perinatal infection), as well as temporal differences in triggering events during lifetime, could then lead to MS. ${ }^{49,51}$

The emerging concept involving HERVs in human medicine $^{21}$ highlights the importance such gene-environment interactions in a number of multifactorial diseases with poorly understood etiology, ${ }^{84}$ including cancer. ${ }^{85}$

\section{Conflict of Interest}

Hervé Perron, Raphaël Faucard, Alexandra Madeira, Ingrid Burgelin, Guillaume Ollagnier, and Corrine Bernard are employed by Geneuro. All other authors declare no conflict of interest.
Acknowledgements. We express all our thanks to: Emmanuelle Abadie and Jean-Romain Richard for their technical assistance; the patients for participating in this study; the Plateforme de Ressources Biologiques, Groupe Hospitalier Albert-Chenevier-Henri Mondor, AP-HP, Créteil, France. We are also very thankful to Dr Faith Dickerson, for reviewing the manuscript. This work was supported by the Institut National pour la Recherche Médicale (INSERM), France; the Assistance Publique des Hôpitaux de Paris (AP-HP), France; the Agence Nationale pour la Recherche (ANR-Project V.I.P.), France and the Réseau Thématique de Recherche et de Soin en Santé Mentale, Fondation FondaMental, France.

1. Van Winkel R, Esquivel G, Kenis G, Wichers M, Collip D, Peerbooms $O$ et al. REVIEW: genome-wide findings in schizophrenia and the role of gene-environment interplay. CNS Neurosci Ther 2010; 16: e185-e192.

2. Lichtenstein P, Yip BH, Bjork C, Pawitan Y, Cannon TD, Sullivan PF et al. Common genetic determinants of schizophrenia and bipolar disorder in Swedish families: a populationbased study. Lancet 2009; 373: 234-239.

3. Brown AS, Derkits EJ. Prenatal infection and schizophrenia: a review of epidemiologic and translational studies. Am J Psychiatry 2010; 167: 261-280.

4. Pearce BD, Kruszon-Moran D, Jones JL. The relationship between Toxoplasma Gondii infection and mood disorders in the third National Health and Nutrition Survey. Biol Psychiatry 2012; 72: 290-295.

5. Mortensen PB, Norgaard-Pedersen B, Waltoft BL, Sorensen TL, Hougaard D, Torrey EF et al. Toxoplasma gondii as a risk factor for early-onset schizophrenia: analysis of filter paper blood samples obtained at birth. Biol Psychiatry 2007; 61: 688-693.

6. Arias I, Sorlozano A, Villegas E, Luna Jde D, McKenney K, Cervilla J et al. Infectious agents associated with schizophrenia: a meta-analysis. Schizophr Res 2012; 136: 128-136.

7. Yolken RH, Torrey EF. Are some cases of psychosis caused by microbial agents? a review of the evidence. Mol Psychiatry 2008; 13: 470-479.

8. Dickerson F, Stallings C, Origoni A, Vaughan C, Khushalani S, Yolken R. Additive effects of elevated C-reactive protein and exposure to Herpes Simplex Virus type 1 on cognitive impairment in individuals with schizophrenia. Schizophr Res 2012; 134: 83-88.

9. Okusaga O, Langenberg P, Sleemi A, Vaswani D, Giegling I, Hartmann AM et al. Toxoplasma gondii antibody titers and history of suicide attempts in patients with schizophrenia. Schizophr Res 2011; 133: 150-155.

10. Bechter K. Updating the mild encephalitis hypothesis of schizophrenia. Prog Neuropsychopharmacol Biol Psych 2012 (in press).

11. Stefansson H, Ophoff RA, Steinberg S, Andreassen OA, Cichon S, Rujescu D et al. Common variants conferring risk of schizophrenia. Nature 2009; 460: 744-747.

12. Figueiredo TC, de Oliveira JR. Reconsidering the association between the major histocompatibility complex and bipolar disorder. J Mol Neurosci 2012; 47: 26-30.

13. Jia $\mathrm{P}$, Wang L, Fanous $\mathrm{AH}$, Chen $\mathrm{X}$, Kendler KS, Zhao Z. A bias-reducing pathway enrichment analysis of genome-wide association data confirmed association of the MHC region with schizophrenia. J Med Genet 2012; 49: 96-103.

14. Shi J, Levinson DF, Duan J, Sanders AR, Zheng Y, Pe'er I et al. Common variants on chromosome 6p22.1 are associated with schizophrenia. Nature 2009; 460: 753-757.

15. Schulze TG, Akula N, Breuer R, Steele J, Nalls MA, Singleton AB et al. Molecular genetic overlap in bipolar disorder, schizophrenia, and major depressive disorder. World J Biol Psychiatry 2012; doi:10.3109/15622975.2012.662282 (e-pub ahead of print).

16. Lee KW, Woon PS, Teo YY, Sim K. Genome wide association studies (GWAS) and copy number variation (CNV) studies of the major psychoses: what have we learnt? Neurosci Biobehav Rev 2012; 36: 556-571.

17. Williams HJ, Norton N, Dwyer S, Moskvina V, Nikolov I, Carroll L et al. Fine mapping of ZNF804A and genome-wide significant evidence for its involvement in schizophrenia and bipolar disorder. Mol Psychiatry 2011; 16: 429-441.

18. Buizer-Voskamp JE, Muntjewerff JW, Strengman E, Sabatti C, Stefansson H, Vorstman JA et al. Genome-wide analysis shows increased frequency of copy number variation deletions in Dutch schizophrenia patients. Biol Psychiatry 2011; 70: 655-662.

19. Flomen RH, Collier DA, Osborne S, Munro J, Breen G St, Clair D et al. Association study of CHRFAM7A copy number and 2bp deletion polymorphisms with schizophrenia and bipolar affective disorder. Am J Med Genet B Neuropsychiatr Genet 2006; 141B: $571-575$.

20. Ingason A, Kirov G, Giegling I, Hansen T, Isles AR, Jakobsen KD et al. Maternally derived microduplications at 15q11-q13: implication of imprinted genes in psychotic illness. Am J Psychiatry 2011; 168: 408-417.

21. Feschotte $C$, Gilbert C. Endogenous viruses: insights into viral evolution and impact on host biology. Nat Rev Genet 2012; 13: 283-296.

22. Huang W, Li S, Hu Y, Yu H, Luo F, Zhang Q et al. Implication of the env gene of the Human Endogenous Retrovirus W family in the expression of BDNF and DRD3 and development of recent-onset schizophrenia. Schizophr Bull 2011; 37: 988-1000.

23. Deb-Rinker P, Klempan TA, O'Reilly RL, Torrey EF, Singh SM. Molecular characterization of a MSRV-like sequence identified by RDA from monozygotic twin pairs discordant for schizophrenia. Genomics 1999; 61: 133-144. 
24. Frank O, Giehl M, Zheng C, Hehlmann R, Leib-Mosch C, Seifarth W. Human endogenous retrovirus expression profiles in samples from brains of patients with schizophrenia and bipolar disorders. J Virol 2005; 79: 10890-10901.

25. Huang W, Li S, Hu Y, Yu H, Luo F, Zhang $Q$ et al. Implication of the env gene of the human endogenous retrovirus $W$ family in the expression of BDNF and DRD3 and development of recent-onset schizophrenia. Schizophr Bull 2011; 37: 988-1000.

26. Huang WJ, Liu ZC, Wei W, Wang GH, Wu JG, Zhu F. Human endogenous retroviral pol RNA and protein detected and identified in the blood of individuals with schizophrenia Schizophr Res 2006; 83: 193-199.

27. Karlsson H, Bachmann S, Schroder J, McArthur J, Torrey EF, Yolken RH. Retroviral RNA identified in the cerebrospinal fluids and brains of individuals with schizophrenia. Proc Natl Acad Sci USA 2001; 98: 4634-4639.

28. Karlsson H, Schroder J, Bachmann S, Bottmer C, Yolken RH. HERV-W-related RNA detected in plasma from individuals with recent-onset schizophrenia or schizoaffective disorder. Mol Psychiatry 2004; 9: 12-13.

29. Lillehoj EP, Ford GM, Bachmann S, Schroder J, Torrey EF, Yolken RH. Serum antibodies reactive with non-human primate retroviruses identified in acute onset schizophrenia. J Neurovirol 2000; 6: 492-497.

30. Perron H, Mekaoui L, Bernard C, Veas F, Stefas I, Leboyer M. Endogenous retrovirus type W GAG and envelope protein antigenemia in serum of schizophrenic patients. Biol Psychiatry 2008; 64: 1019-1023.

31. Yao Y, Schroder J, Nellaker C, Bottmer C, Bachmann S, Yolken RH et al. Elevated levels of human endogenous retrovirus-W transcripts in blood cells from patients with first episode schizophrenia. Genes Brain Behav 2008; 7: 103-112.

32. Yolken RH, Karlsson H, Yee F, Johnston-Wilson NL, Torrey EF. Endogenous retroviruses and schizophrenia. Brain Res Brain Res Rev 2000; 31: 193-199.

33. Perron H, Garson JA, Bedin F, Beseme F, Paranhos-Baccala G, Komurian-Pradel F et al. Molecular identification of a novel retrovirus repeatedly isolated from patients with multiple sclerosis. The Collaborative Research Group on multiple sclerosis. Proc Natl Acad Sci USA 1997; 94: 7583-7588.

34. Blond JL, Beseme F, Duret L, Bouton O, Bedin F, Perron $\mathrm{H}$ et al. Molecular characterization and placental expression of HERV-W, a new human endogenous retrovirus family. J Virol 1999; 73: 1175-1185.

35. Kim HS, Takenaka O, Crow TJ. Isolation and phylogeny of endogenous retrovirus sequences belonging to the HERV-W family in primates. J Gen Virol 1999; 80(Pt 10): 2613-2619.

36. Komurian-Pradel F, Paranhos-Baccala G, Bedin F, Ounanian-Paraz A, Sodoyer M, Ott C et al. Molecular cloning and characterization of MSRV-related sequences associated with retroviruslike particles. Virology 1999; 260: 1-9.

37. Perron $\mathrm{H}$, Perin JP, Rieger F, Alliel PM. Particle-associated retroviral RNA and tandem RGH/HERV-W copies on human chromosome 7q: possible components of a 'chainreaction' triggered by infectious agents in multiple sclerosis? J Neurovirol 2000; 6(Suppl 2): S67-S75.

38. Bruno S, Cercignani M, Ron MA. White matter abnormalities in bipolar disorder: a voxelbased diffusion tensor imaging study. Bipolar Disord 2008; 10: 460-468.

39. El-Badri SM, Cousins DA, Parker S, Ashton HC, McAllister VL, Ferrier IN et al. Magnetic resonance imaging abnormalities in young euthymic patients with bipolar affective disorder Br J Psychiatry 2006; 189: 81-82.

40. Gonenc A, Frazier JA, Crowley DJ, Moore CM. Combined diffusion tensor imaging and transverse relaxometry in early-onset bipolar disorder. J Am Acad Child Adolesc Psychiatry 2010; 49: 1260-1268.

41. Heng S, Song AW, Sim K. White matter abnormalities in bipolar disorder: insights from diffusion tensor imaging studies. J Neural Transm 2010; 117: 639-654.

42. Mahon K, Wu J, Malhotra AK, Burdick KE, DeRosse P, Ardekani BA et al. A voxel-based diffusion tensor imaging study of white matter in bipolar disorder. Neuropsychopharmacology 2009; 34: 1590-1600.

43. Wang F, Kalmar JH, Edmiston E, Chepenik LG, Bhagwagar Z, Spencer L et al. Abnormal corpus callosum integrity in bipolar disorder: a diffusion tensor imaging study. Biol Psychiatry 2008; 64: 730-733.

44. Stevens JR. Neuropathology of schizophrenia. Arch Gen Psychiatry 1982; 39: 1131-1139.

45. Perron H, Germi R, Bernard C, Garcia-Montojo M, Deluen C, Farinelli L. Human Endogenous Retrovirus type $\mathrm{W}$ envelope expression in blood and brain cells provides new insights into multiple sclerosis disease. Mult Scler advance online publication, 30 March 2012; doi:10.1177/1352458512441381.

46. Romanish MT, Cohen CJ, Mager DL. Potential mechanisms of endogenous retroviralmediated genomic instability in human cancer. Semin Cancer Biol 2010; 20: 246-253.

47. Belshaw R, Katzourakis A, Paces J, Burt A, Tristem M. High copy number in human endogenous retrovirus families is associated with copying mechanisms in addition to reinfection. Mol Biol Evol 2005; 22: 814-817.

48. Nellaker $\mathrm{C}, \mathrm{Yao} \mathrm{Y}$, Jones-Brando L, Mallet F, Yolken RH, Karlsson $\mathrm{H}$. Transactivation of elements in the human endogenous retrovirus $\mathrm{W}$ family by viral infection. Retrovirology 2006; 3: 44.

49. Perron $\mathrm{H}$, Lang $\mathrm{A}$. The human endogenous retrovirus link between genes and environment in multiple sclerosis and in multifactorial diseases associating neuroinflammation. Clin Rev Allergy Immunol 2010; 39: 51-61.

50. Dawkins R, Leelayuwat C, Gaudieri S, Tay G, Hui J, Cattley S et al. Genomics of the major histocompatibility complex: haplotypes, duplication, retroviruses and disease. Immunol Rev 1999; 167: 275-304.
51. Leboyer M, Tamouza R, Charron D, Faucard R, Perron H. Human endogenous retrovirus type W (HERV-W) in schizophrenia: a new avenue of research at the gene-environment interface. World J Biol Psychiatry advance online publication, 22 September 2011; doi:10.3109/15622975.2010.601760.

52. Arias I, Sorlozano A, Villegas E, de Dios Luna J, McKenney K, Cervilla J et al. Infectious agents associated with schizophrenia: a meta-analysis. Schizophr Res 2012; 136: 128-136.

53. Doorduin J, de Vries EF, Willemsen AT, de Groot JC, Dierckx RA, Klein HC. Neuroinflammation in schizophrenia-related psychosis: a PET study. J Nucl Med 2009; 50: $1801-1807$.

54. De Peri L, Crescini A, Deste G, Fusar-Poli P, Sacchetti E, Vita A. Brain structural abnormalities at the onset of schizophrenia and bipolar disorder: a metaanalysis of controlled magnetic resonance imaging studies. Curr Pharm Des 2012; 18: 486-494.

55. Mirsattari SM, Johnston JB, McKenna R, Del Bigio MR, Orr P, Ross RT et al. Aboriginals with multiple sclerosis: HLA types and predominance of neuromyelitis optica. Neurology 2001; 56: 317-323

56. Wouters D, van Schouwenburg P, van der Horst A, de Boer M, Schooneman D, Kuijpers TW et al. High-throughput analysis of the C4 polymorphism by a combination of MLPA and isotype-specific ELISA's. Mol Immunol 2009; 46: 592-600.

57. Griffiths DJ. Endogenous retroviruses in the human genome sequence. Genome Biol 2001; 2: 1017.

58. Bannert N, Kurth $\mathrm{R}$. Retroelements and the human genome: new perspectives on an old relation. Proc Natl Acad Sci USA 2004; 101(Suppl 2): 14572-14579.

59. Challem JJ, Taylor EW. Retroviruses, ascorbate, and mutations, in the evolution of Homo sapiens. Free Radic Biol Med 1998; 25: 130-132.

60. Schulz WA, Steinhoff C, Florl AR. Methylation of endogenous human retroelements in health and disease. Curr Top Microbiol Immunol 2006; 310: 211-250.

61. Perron H, Bernard C, Bertrand JB, Lang AB, Popa I, Sanhadji K et al. Endogenous retroviral genes, Herpesviruses and gender in multiple sclerosis. J Neurol Sci 2009; 286: 65-72.

62. Frank O, Jones-Brando L, Leib-Mosch C, Yolken R, Seifarth W. Altered transcriptional activity of human endogenous retroviruses in neuroepithelial cells after infection with Toxoplasma gondii. J Infect Dis 2006; 194: 1447-1449.

63. Haahr S, Munch M. The association between multiple sclerosis and infection with EpsteinBarr virus and retrovirus. J Neurovirol 2000; 6(Suppl 2): S76-S79.

64. Perron H, Suh M, Lalande B, Gratacap B, Laurent A, Stoebner P et al. Herpes simplex virus ICPO and ICP4 immediate early proteins strongly enhance expression of a retrovirus harboured by a leptomeningeal cell line from a patient with multiple sclerosis. J Gen Virol 1993; 74(Pt 1): 65-72

65. Ruprecht K, Obojes K, Wengel V, Gronen F, Kim KS, Perron H et al. Regulation of human endogenous retrovirus $\mathrm{W}$ protein expression by herpes simplex virus type 1 : implications for multiple sclerosis. J Neurovirol 2006; 12: 65-71.

66. Rolland A, Jouvin-Marche E, Viret C, Faure M, Perron H, Marche PN. The envelope protein of a human endogenous retrovirus-W family activates innate immunity through CD14/TLR4 and promotes Th1-like responses. J Immunol 2006; 176: 7636-7644.

67. Mameli G, Poddighe L, Astone V, Delogu G, Arru G, Sotgiu S et al. Novel reliable real-time PCR for differential detection of MSRVenv and syncytin-1 in RNA and DNA from patients with multiple sclerosis. J Virol Methods 2009; 161: 98-106.

68. Nurnberger JI Jr, Blehar MC, Kaufmann CA, York-Cooler C, Simpson SG, HarkavyFriedman $\mathrm{J}$ et al. Diagnostic interview for genetic studies. Rationale, unique features, and training. NIMH genetics initiative. Arch Gen Psychiatry 1994; 51: 849-859; discussion 863-844

69. Kay SR, Fiszbein A, Opler LA. The positive and negative syndrome scale (PANSS) for schizophrenia. Schizophr Bull 1987; 13: 261-276.

70. Addington D, Addington J, Maticka-Tyndale E. Assessing depression in schizophrenia: the Calgary Depression Scale. Br J Psychiatry Suppl 1993; 22: 39-44.

71. Young RC, Biggs JT, Ziegler VE, Meyer DA. A rating scale for mania: reliability, validity and sensitivity. Br J Psychiatry 1978; 133: 429-435.

72. Montgomery SA, Asberg M. A new depression scale designed to be sensitive to change. Br J Psychiatry 1979; 134: 382-389.

73. Dickerson FB, Boronow JJ, Stallings C, Origoni AE, Ruslanova I, Yolken RH. Association of serum antibodies to herpes simplex virus 1 with cognitive deficits in individuals with schizophrenia. Arch Gen Psychiatry 2003; 60: 466-472.

74. Diem O, Schaffner M, Seifarth W, Leib-Mosch C. Influence of antipsychotic drugs on human endogenous retrovirus (HERV) transcription in brain cells. PLOS One 2012; 7: e30054.

75. Perron H, Jouvin-Marche E, Michel M, Ounanian-Paraz A, Camelo S, Dumon A et al. Multiple sclerosis retrovirus particles and recombinant envelope trigger an abnormal immune response in vitro, by inducing polyclonal Vbeta16 T-lymphocyte activation. Virology 2001; 287: 321-332.

76. Anderson JL, Carlquist JF, Muhlestein JB, Horne BD, Elmer SP. Evaluation of C-reactive protein, an inflammatory marker, and infectious serology as risk factors for coronary artery disease and myocardial infarction. J Am Coll Cardiol 1998; 32: 35-41.

77. Weng CM, Chou CH, Huang YY, Lin CC, Liu YW, Tsai WC. Increased C-reactive protein is associated with future development of diabetes mellitus in essential hypertensive patients. Heart Vessels 2010; 25: 386-391. 
78. Torrey EF, Bartko JJ, Yolken RH. Toxoplasma gondii and other risk factors for Schizophrenia: an update. Schizophr Bull 2012; 38: 642-647.

79. Bliek J, Maas SM, Ruijter JM, Hennekam RC, Alders M, Westerveld A et al. Increased tumour risk for BWS patients correlates with aberrant $\mathrm{H} 19$ and not KCNQ1OT1 methylation: occurrence of KCNQ1OT1 hypomethylation in familial cases of BWS. Hum Mol Genet 2001; 10: 467-476

80. Badal V, Chuang LS, Tan EH, Badal S, Villa LL, Wheeler CM et al. CpG methylation of human papillomavirus type 16 DNA in cervical cancer cell lines and in clinical specimens: genomic hypomethylation correlates with carcinogenic progression. J Virol 2003; 77: 62276234.

81. Samuelsson J, Alonso S, Ruiz-Larroya T, Cheung TH, Wong YF, Perucho M. Frequent somatic demethylation of RAPGEF1/C3G intronic sequences in gastrointestinal and gynecological cancer. Int J Oncol 2011; 38: 1575-1577.

82. Zvetkova I, Apedaile A, Ramsahoye B, Mermoud JE, Crompton LA, John R et al. Global hypomethylation of the genome in XX embryonic stem cells. Nat Genet 2005; 37 : 1274-1279.
83. Dolei A, Perron $\mathrm{H}$. The multiple sclerosis-associated retrovirus and its HERV-W endogenous family: a biological interface between virology, genetics, and immunology in human physiology and disease. J Neurovirol 2009; 15: 4-13.

84. Douville R, Liu J, Rothstein J, Nath A. Identification of active loci of a human endogenous retrovirus in neurons of patients with amyotrophic lateral sclerosis. Ann Neurol 2011; 69: 141-151.

85. Engel ME, Hiebert SW. The enemy within: dormant retroviruses awaken. Nat Med 2010; 16: $517-518$

(c) Translational Psychiatry is an open-access journal SOMERIHHISRESERVED published by Nature Publishing Group. This work is licensed under the Creative Commons Attribution-NonCommercial-No Derivative Works 3.0 Unported License. To view a copy of this license, visit http://creativecommons.org/licenses/by-nc-nd/3.0/

Supplementary Information accompanies the paper on the Translational Psychiatry website (http://www.nature.com/tp) 